\title{
Primary Small Cell Carcinoma of the Pancreas Presenting as Lower Extremity Swelling
}

\author{
Phuong Ngo ${ }^{\text {a }}$, Jenna Zauk ${ }^{\mathrm{b}}$, John Beal ${ }^{\mathrm{c}, ~ e}$, Ethan Wasserman ${ }^{\mathrm{d}}$
}

\begin{abstract}
Primary small cell carcinoma of the pancreas is a rare malignancy with a very poor prognosis. There have been fewer than 50 cases reported with the majority of patients presenting with abdominal symptoms. We report a case of a patient with a psychiatric history who presented with lower extremity swelling. Further testing revealed metastatic small cell carcinoma of the pancreas, and she died within 2 weeks of diagnosis. Our case highlights the need for better recognition of this malignancy to hasten diagnosis and treatment.
\end{abstract}

Keywords: Primary small cell carcinoma; Pancreas

\section{Introduction}

Small cell carcinoma typically arises in the lung but can also originate in extrapulmonary sites though this is extremely rare. These tumors have been found in the salivary glands, larynx, esophagus, stomach, colon, rectum, gallbladder, urinary bladder, prostate, cervix and the skin. Increasingly, these malignancies have also been described as arising from the pancreas. Though these cancers have shown to be very aggressive, various treatment regimens have had an impact on progression free survival, thus necessitating the need for earlier diagnosis.

\section{Case Report}

A 64-year-old obese, Caucasian female presented to the emer-

Manuscript submitted May 14, 2018, accepted June 12, 2018

aDepartment of Hematology and Oncology, University of Louisville, Louisville, KY, USA

bDepartment of Anesthesiology, University of Toledo Medical Center, Toledo, $\mathrm{OH}, \mathrm{USA}$

'Department of Medicine, Jersey Shore University Medical Center, Neptune, NJ, USA

dDepartment of Hematology and Oncology, Jersey Shore University Medical Center, Neptune, NJ, USA

${ }^{\mathrm{e} C o r r e s p o n d i n g ~ A u t h o r: ~ J o h n ~ B e a l, ~ D e p a r t m e n t ~ o f ~ M e d i c i n e, ~ J e r s e y ~ S h o r e ~}$ University Medical Center, 1945 State Route 33, Neptune, NJ 07753, USA.

Email: John.Beal@hackensackmeridian.org

doi: https://doi.org/10.14740/jmc3098w gency room with progressive bilateral lower extremity swelling for 2 weeks that affected her ability to walk. She had presented to her primary care physician when symptoms began and was started on spironolactone, but the edema persisted. She also reported weight gain, increased thirst, increased appetite, mild shortness of breath, and diffuse bruising. She denied nausea, vomiting, abdominal pain, or changes in bowel movements. Her medical history included schizoaffective disorder and psychogenic polydipsia. She had no history of tobacco, alcohol or substance abuse. Family history was significant for a mother with breast cancer, father with lung cancer, paternal aunt with uterine and ovarian cancer and a paternal cousin with possible ovarian or pancreatic cancer. Vital signs were within normal limits and physical exam was significant only for pitting edema in the lower extremities. Complete metabolic panel showed sodium $126 \mathrm{mmol} / \mathrm{L}$, potassium $3.4 \mathrm{mmol} / \mathrm{L}$, and creatinine $1.37 \mathrm{mg} / \mathrm{dL}$ from a baseline of $0.9 \mathrm{mg} / \mathrm{dL}$, and a complete blood count was within normal limits. Liver enzymes were elevated with alkaline phosphatase $202 \mathrm{U} / \mathrm{L}$, aspartate aminotransferase $107 \mathrm{U} / \mathrm{L}$, alanine aminotransferase $148 \mathrm{U} / \mathrm{L}$ but normal total bilirubin. 2D echocardiogram showed a $>$ $55 \%$ ejection fraction without systolic dysfunction. She was fluid restricted and given furosemide with improvement in the edema. Renal ultrasound showed a $15 \mathrm{~cm}$ mass arising from the lower pole of the left kidney, but a follow-up computed tomography (CT) of the abdomen and pelvis revealed that this mass was actually arising from the pancreas with displacement of surrounding structures, splenic vein thrombosis with short gastric varices, and innumerable hepatic lesions (Fig. 1). CT chest was negative for pulmonary lesions and magnetic resonance imaging (MRI) of the brain was negative. Cancer antigen (CA) 125, CA 19-9, carcinoembryonic antigen (CEA) and alpha-fetoprotein (AFP) were all elevated. Liver biopsy was consistent with small cell carcinoma of pancreatic origin. She was started on cisplatin and etoposide, and appeared to tolerate the treatment well but ultimately passed away from unknown causes within the week.

\section{Discussion}

Small cell carcinoma of the pancreas is becoming more recognized but still remains a rare malignancy with a variety of presenting symptoms. The majority of patients present with nausea, vomiting, abdominal discomfort, weight loss, and jaundice though there have been a few cases with unusual presentations such as hyperkalemia [1] and hypercalcemia [2]. 


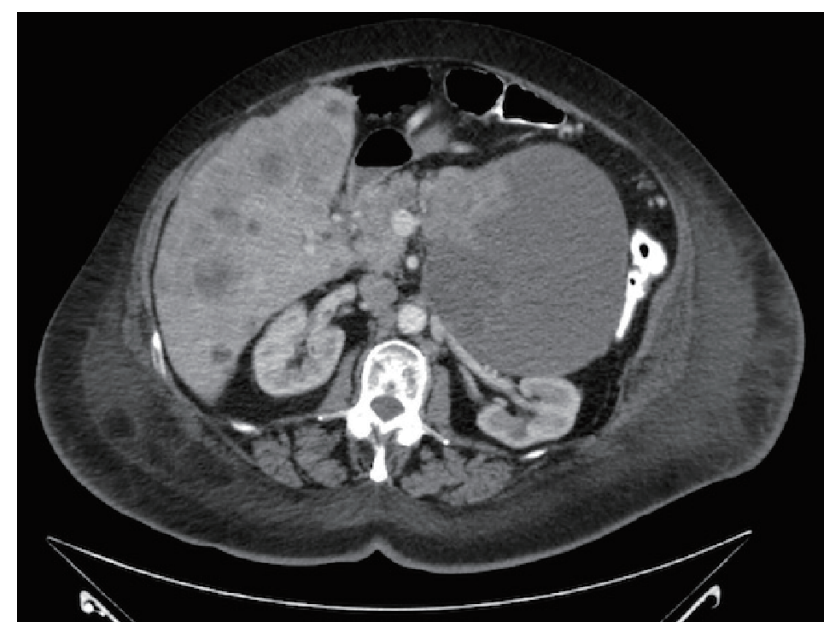

Figure 1. CT scan of abdomen and pelvis: large cystic and solid mass arising from the tail of the pancreas, measuring approximately $13.5 \times$ $12.7 \mathrm{~cm}$; liver is calcified with innumerable metastatic deposits.

One patient presented with progressive vision loss due to orbital metastases from small cell pancreatic cancer [3]. Two cases have described associated paraneoplastic syndromes similar to those well recognized in small cell lung cancer [1]. Our patient passed away before further workup, but her unusual symptoms further emphasize the often atypical presentations of this malignancy.

Treatment guidelines are also difficult to establish due to the paucity of studies. SCC of the pancreas was first described as a series of five cases in 1981. These patients were managed symptomatically and had a survival rate of $1-2$ months $[4,5]$. A surgical series later identified six patients at Johns Hopkins and the Mayo Clinic who had surgical resection of a pancreatic small cell carcinoma with five of these patients receiving adjuvant treatment. Five patients received combined adjuvant radiation with concurrent cisplatin and etoposide and one patient received neoadjuvant 5-fluorouracil, adriamycin and mitomycin. Survival ranged from 9 - 173 months with median survival of 20 months [5]. Since then, other cases have also described treatment with platinum and etoposide with the longest survival reported as 56 months [6].

Though pancreatic small cell remains an aggressive malignancy with a poor prognosis, various combinations of treatment including surgical resection, radiation and chemotherapy have prolonged survival in these patients since it was first described. Earlier recognition is therefore pivotal. Our patient ultimately passed from her malignancy, but we hope to increase awareness of the atypical signs and symptoms of this disease, the possibility of associated paraneoplastic syndromes and the necessity of starting treatment as soon as possible.

\section{References}

1. Caesar J, Jordan M, Kumar P, Gray C. Primary small cell carcinoma of the pancreas presenting with likely paraneoplastic features. ACG Case Rep J. 2016;3(3):190-192.

2. Hobbs RD, Stewart AF, Ravin ND, Carter D. Hypercalcemia in small cell carcinoma of the pancreas. Cancer. 1984;53(7):1552-1554.

3. Rong AJ, Choi CJ, Ghassibi MP, Dubovy SR, Johnson TE. Orbital metastatic small cell carcinoma of the pancreas with optic nerve compression. Can J Ophthalmol. 2018;53(1):e22-e24.

4. Reyes CV, Wang T. Undifferentiated small cell carcinoma of the pancreas: a report of five cases. Cancer. 1981;47(10):2500-2502.

5. Wang D, Rong Y, Wu W, Jin D. Primary small cell carcinoma of the pancreas: rare type of pancreatic cancer and review of the literatures. World J Surg Oncol. 2012;10:32.

6. Winter JM, Narang AK, Mansfield AS, Herman JM, Cameron JL, Laheru D, Eckhauser FE, et al. Resectable pancreatic small cell carcinoma. Rare Tumors. 2011;3(1):e5. 